\title{
A simple proof of Sen's possibility theorem on majority decisions
}

\author{
Christian Elsholtz and Christian List \\ Christian Elsholtz studied mathematics at the Technical University of Darmstadt, \\ where he received his Ph.D. He worked at the University of Stuttgart and completed \\ his habilitation at the Technical University of Clausthal. He is currently a lecturer in \\ pure mathematics at Royal Holloway University of London. Much of his work is on \\ gaps between prime numbers. \\ Christian List holds a B.A. in mathematics and philosophy, and an M.Phil. and a \\ D.Phil. in politics from the University of Oxford. After visiting positions at the \\ Australian National University in Canberra, at Harvard University and MIT, he was a \\ postdoctoral research fellow at Nuffield College in Oxford. He is currently a lecturer \\ in political science at the London School of Economics. His research area lies at the \\ intersection between political philosophy and mathematical decision theory.
}

\section{Introduction}

Condorcet's paradox shows that pairwise majority voting over three or more candidates can lead to cyclical majority preferences, even when the preferences of individual voters are transitive: Suppose there are three voters, labelled 1, 2 and 3, and three candidates, labelled $x_{1}, x_{2}$ and $x_{3}$ with the following preferences (the symbol ' $\succ$ ' means 'is strictly

Das Condorcetsche Wahlparadoxon zeigt, dass paarweise Mehrheitsvergleiche zwischen drei oder mehr Kandidaten einen ungewollten Zykel ergeben können: nämlich, dass eine Mehrheit Kandidat $A$ vor $B$ bevorzugt, eine Mehrheit $B$ vor $C$, aber dennoch eine Mehrheit für $C$ vor $A$ stimmt, selbst dann, wenn jeder einzelne Wähler eine nichtzyklische Präferenzliste hat. Mehrheitszykel sind ein in der Politikwissenschaft häufig diskutiertes Phänomen, da sie Fragen zu den Grundlagen der Demokratie aufwerfen. In einer wichtigen Arbeit gab der Wirtschaftsnobelpreisträger Amartya Sen eine hinreichende Bedingung an, die solche Zykel ausschließt. In der vorliegenden Arbeit präsentieren die Autoren einen elementaren Beweis von Sens Satz und diskutieren eine Variante seiner Bedingung. Weiterhin geben sie eine notwendige und hinreichende Bedingung zur Vermeidung von Zykeln an, die zeigt, dass Sens Bedingung nicht in befriedigender Weise weiter verallgemeinert werden kann. 
preferred to'):

Voter 1: $x_{1} \succ x_{2} \succ x_{3}$,

Voter 2: $x_{2} \succ x_{3} \succ x_{1}$,

Voter 3: $x_{3} \succ x_{1} \succ x_{2}$.

Then there are majorities of 2 out of 3 voters for $x_{1} \succ x_{2}$, for $x_{2} \succ x_{3}$ and for $x_{3} \succ x_{1}$. The resulting majority preference ordering is cyclical: $x_{1} \succ x_{2} \succ x_{3} \succ x_{1}$. Cyclical majority preferences (in short: cycles) are democratically undesirable, as they are unsuitable for reaching consistent democratic decisions. A large literature in social choice theory addresses the threat posed by cycles for the functioning of democratic decision mechanisms (for example, Riker [4]). Several sufficient conditions for the avoidance of cycles have been identified. Black [1] showed that, if the $n$-tuple of individual preference orderings across $n$ voters satisfies an appealing condition called single-peakedness, the resulting majority preference ordering will be transitive. Later, other sufficient conditions for transitive majority preference were found, amongst them single-cavedness (Inada [2]), separability into two groups (Inada [2]), and latin-squarelessness (Ward [6]). (See also Section 3 below.)

In a famous paper, Sen [5] generalized these results, showing that a condition that is less demanding than, but implied by, each of these conditions is already sufficient for avoiding cycles. Sen's condition is called triplewise value-restriction. However, Sen's condition and theorem are not intuitively straightforward. This note aims to make the mechanism underlying Sen's result easily accessible, by giving an elementary proof of Sen's theorem, together with a simple reformulation of the condition of triplewise value-restriction. In terms of our reformulation, we also discuss how Sen's condition is logically related to its precursors. Finally, we suggest that, although there is still some logical space between Sen's sufficient condition for the avoidance of cycles and a necessary and sufficient condition, this space may be too narrow to allow an appealing generalization of Sen's condition.

We are grateful to an anonymous referee for comments.

\section{An easy proof of a slightly simplified version of Sen's theorem}

We first prove a slightly simplified variant of Sen's result. Suppose there are $n$ voters, labelled $1,2, \ldots, n$, and $k$ candidates, labelled $x_{1}, x_{2}, \ldots, x_{k}$. To avoid ties under majority voting, we assume that $n$ is odd. And suppose each voter holds a preference ordering over the candidates. We use the notation $x_{1} \succ x_{2}$ to mean that the voter strictly prefers candidate $x_{1}$ to candidate $x_{2}$. Each voter's preference ordering is assumed to be complete and transitive.

Completeness. For any two candidates $x_{1}, x_{2}$, either $x_{1} \succ x_{2}$ or $x_{2} \succ x_{1}$ (but not both).

Transitivity. For any $x_{1}, x_{2}, x_{3}$, if $x_{1} \succ x_{2}$ and $x_{2} \succ x_{3}$, then $x_{1} \succ x_{3}$.

An $n$-tuple of individual preference orderings across $n$ voters is called a profile of individual preference orderings, in short a profile. A profile is triplewise value-restricted if it satisfies the following property. 
Triplewise value-restriction. For every triple of distinct candidates $x_{1}, x_{2}, x_{3}$, there exists $x_{i} \in\left\{x_{1}, x_{2}, x_{3}\right\}$ and $r \in\{1,2,3\}$ such that no voter ranks $x_{i}$ as his or her $r$-th preference among $x_{1}, x_{2}, x_{3}$.

Theorem 1 (Sen 1966) For every profile satisfying triplewise value-restriction, pairwise majority voting generates a transitive (hence acylic) majority preference ordering.

Sen's original result allows voters to be indifferent between two or more candidates. In this section, we assume that voters always order candidates in a strict ranking. In Section 4, we explain how our method can be used to prove Sen's result in full generality.

Our proof is in three steps. In a first step, we identify a condition that is sufficient for avoiding cycles over triples of alternatives. In a second step, we show that, if this condition holds for every triple of alternatives, this is sufficient for the avoidance of any cycles. In a third step, we show that our condition (applied to every triple of alternatives) is logically equivalent to triplewise value-restriction, as stated above.

Step 1. Consider three candidates, $x_{1}, x_{2}, x_{3}$. There are six logically possible strict preference orderings over $x_{1}, x_{2}, x_{3}$ :

$$
\begin{aligned}
& \text { 1: } x_{1} \succ x_{2} \succ x_{3}, \quad 2: x_{1} \succ x_{3} \succ x_{2}, \quad 3: x_{2} \succ x_{1} \succ x_{3} \text {, } \\
& \text { 4: } x_{2} \succ x_{3} \succ x_{1}, 5: x_{3} \succ x_{1} \succ x_{2}, 6: x_{3} \succ x_{2} \succ x_{1} \text {. }
\end{aligned}
$$

Let $a_{h i j}$ denote the number of voters holding the preference ordering $x_{h} \succ x_{i} \succ x_{j}\left(a_{h i j}\right.$ is a non-negative integer). A preference ordering can be represented as a matrix $M=\left(m_{i j}\right)$, where

$$
m_{i j}= \begin{cases}1 & \text { if } x_{i} \succ x_{j} \\ 0 & \text { otherwise }\end{cases}
$$

The six orderings above thus correspond to the following matrices:

$$
\begin{array}{lll}
M_{123}=\left(\begin{array}{lll}
0 & 1 & 1 \\
0 & 0 & 1 \\
0 & 0 & 0
\end{array}\right), & M_{132}=\left(\begin{array}{lll}
0 & 1 & 1 \\
0 & 0 & 0 \\
0 & 1 & 0
\end{array}\right), & M_{213}=\left(\begin{array}{lll}
0 & 0 & 1 \\
1 & 0 & 1 \\
0 & 0 & 0
\end{array}\right), \\
M_{231}=\left(\begin{array}{lll}
0 & 0 & 0 \\
1 & 0 & 1 \\
1 & 0 & 0
\end{array}\right), & M_{312}=\left(\begin{array}{lll}
0 & 1 & 0 \\
0 & 0 & 0 \\
1 & 1 & 0
\end{array}\right), & M_{321}=\left(\begin{array}{lll}
0 & 0 & 0 \\
1 & 0 & 0 \\
1 & 1 & 0
\end{array}\right) .
\end{array}
$$

Pairwise majority voting corresponds to the following weighted sum:

$$
\begin{aligned}
S & =a_{123} M_{123}+a_{132} M_{132}+a_{213} M_{213}+a_{231} M_{231}+a_{312} M_{312}+a_{321} M_{321} \\
& =\left(\begin{array}{ccc}
0 & a_{123}+a_{132}+a_{312} & a_{123}+a_{132}+a_{213} \\
a_{213}+a_{231}+a_{321} & 0 & a_{123}+a_{213}+a_{231} \\
a_{231}+a_{312}+a_{321} & a_{132}+a_{312}+a_{321} & 0
\end{array}\right) .
\end{aligned}
$$

$S=\left(s_{i j}\right)$ induces a majority preference ordering defined as follows. For each $i, j, x_{i} \succ x_{j}$ holds if and only if $s_{i j}>s_{j i}$. 
When can a cycle occur under pairwise majority voting? There are two logically possible cycles: $x_{1} \succ x_{2} \succ x_{3} \succ x_{1}$ and $x_{1} \succ x_{3} \succ x_{2} \succ x_{1}$.

Suppose we have a majority cycle of the first type, $x_{1} \succ x_{2} \succ x_{3} \succ x_{1}$.

$$
\begin{array}{lll}
x_{1} \succ x_{2} \text { means } s_{12}>s_{21}, & \text { i.e. } & a_{123}+a_{132}+a_{312}>a_{213}+a_{231}+a_{321} . \\
x_{2} \succ x_{3} \text { means } s_{23}>s_{32}, & \text { i.e. } & a_{123}+a_{213}+a_{231}>a_{132}+a_{312}+a_{321} . \\
x_{3} \succ x_{1} \text { means } s_{31}>s_{13}, & \text { i.e. } & a_{231}+a_{312}+a_{321}>a_{123}+a_{132}+a_{213} .
\end{array}
$$

We now add pairs of these inequalities.

$$
\begin{aligned}
& (1)+(2) \text { implies } a_{123}>a_{321}, \\
& (1)+(3) \text { implies } a_{312}>a_{213}, \\
& (2)+(3) \text { implies } a_{231}>a_{132} .
\end{aligned}
$$

Analogously, a majority cycle of the second type, $x_{1} \succ x_{3} \succ x_{2} \succ x_{1}$, implies the reverse inequalities, $a_{321}>a_{123}, a_{213}>a_{312}$ and $a_{132}>a_{231}$. Hence, we have the following lemma:

Lemma 1 If there is a majority cycle over $x_{1}, x_{2}, x_{3}$, then $\left(a_{123}>a_{321}\right.$ and $a_{312}>a_{213}$ and $\left.a_{231}>a_{132}\right)$ or $\left(a_{321}>a_{123}\right.$ and $a_{213}>a_{312}$ and $\left.a_{132}>a_{231}\right)$.

An obvious corollary of Lemma 1 is the following:

Lemma 2 If $\left(a_{123} \leq a_{321}\right.$ or $a_{312} \leq a_{213}$ or $\left.a_{231} \leq a_{132}\right)$ and $\left(a_{321} \leq a_{123}\right.$ or $a_{213} \leq a_{312}$ or $\left.a_{132} \leq a_{231}\right)$, then there is no majority cycle over $x_{1}, x_{2}, x_{3}$.

We can infer a corollary of Lemma 2 which is suitable for proving Sen's theorem.

Lemma 3 If $\left(a_{123}=0\right.$ or $a_{312}=0$ or $\left.a_{231}=0\right)$ and $\left(a_{321}=0\right.$ or $a_{213}=0$ or $\left.a_{132}=0\right)$, then there is no majority cycle over $x_{1}, x_{2}, x_{3}$.

Step 2. Now suppose there are $k$ candidates.

Lemma 4 (Standard result) If there is a cycle over $m$ candidates $(3 \leq m \leq k)$ in the majority preference ordering, then there is also a cycle over three candidates in that ordering.

Proof. Suppose there is a cycle over $m$ candidates, $x_{1}, x_{2}, \ldots, x_{m}$, in the majority preference ordering, i.e. $x_{1} \succ x_{2} \succ \ldots \succ x_{m} \succ x_{1}$. We have $x_{1} \succ x_{2}$ and $x_{2} \succ x_{3}$. Either $x_{3} \succ x_{1}$ or $x_{1} \succ x_{3}$. If $x_{3} \succ x_{1}$, we have found a cycle over three candidates, namely $x_{1}$, $x_{2}, x_{3}$. If $x_{1} \succ x_{3}$, we consider $x_{1} \succ x_{3}$ and $x_{3} \succ x_{4}$. Again, either $x_{4} \succ x_{1}$, in which case we have a cycle over $x_{1}, x_{3}, x_{4}$, or $x_{1} \succ x_{4}$. We continue until we reach either a cycle over three candidates, or until we reach $x_{1} \succ x_{m-1}, x_{m-1} \succ x_{m}$. But $x_{m} \succ x_{1}$, and hence we have a cycle over $x_{1}, x_{m-1}, x_{m}$.

Note that any complete strict preference ordering is either transitive or cyclic (where the ordering is cyclic if there exists at least one cycle). Lemma 3 and Lemma 4 imply the following theorem: 
Theorem 2 Suppose, for every triple of distinct candidates $x_{1}, x_{2}, x_{3}$, we have $\left(a_{123}=0\right.$ or $a_{312}=0$ or $\left.a_{231}=0\right)$ and $\left(a_{132}=0\right.$ or $a_{321}=0$ or $\left.a_{213}=0\right)$. Then pairwise majority voting generates a transitive (hence acylic) majority preference ordering.

Step 3. To see that Sen's theorem is an immediate corollary of Theorem 2, we give a simple reformulation of triplewise value-restriction.

Lemma 5 A profile satisfies triplewise value-restriction if and only if, for every triple of distinct candidates $x_{1}, x_{2}, x_{3}:\left(a_{123}=0\right.$ or $a_{312}=0$ or $\left.a_{231}=0\right)$ and $\left(a_{132}=0\right.$ or $a_{321}=0$ or $\left.a_{213}=0\right)$.

Proof. For every triple of distinct candidates $x_{1}, x_{2}, x_{3}$, Sen's condition of triplewise valuerestriction corresponds to a disjunction of nine cases, as detailed in the first two rows of the following table. Each of the nine cases is equivalent to a corresponding case in the third row, and the condition of Lemma 5 is precisely the disjunction of these cases.

\begin{tabular}{|c|c|c||c|c|c||c|c|c|}
\hline$x_{1}$ & $x_{2}$ & $x_{3}$ & $x_{1}$ & $x_{2}$ & $x_{3}$ & $x_{1}$ & $x_{2}$ & $x_{3}$ \\
\hline \multicolumn{3}{|c||}{ is not ranked 1st } & \multicolumn{3}{c||}{ is not ranked 2nd } & \multicolumn{3}{c|}{ is not ranked 3rd } \\
\multicolumn{2}{|c||}{ by any voter if and only if } & \multicolumn{2}{c||}{ by any voter if and only if } & \multicolumn{3}{c|}{ by any voter if and only if } \\
\hline$a_{123}=0$ & $a_{213}=0$ & $a_{312}=0$ & $a_{213}=0$ & $a_{123}=0$ & $a_{132}=0$ & $a_{231}=0$ & $a_{132}=0$ & $a_{123}=0$ \\
$\&$ & $\&$ & $\&$ & $\&$ & $\&$ & $\&$ & $\&$ & $\&$ & $\&$ \\
$a_{132}=0$ & $a_{231}=0$ & $a_{321}=0$ & $a_{312}=0$ & $a_{321}=0$ & $a_{231}=0$ & $a_{321}=0$ & $a_{312}=0$ & $a_{213}=0$ \\
\hline
\end{tabular}

Table 1

\section{Sen's condition and its precursors}

Table 1 is revealing in another respect. Each of the three sets of conditions ( is not ranked 1st', 'is not ranked 2nd', 'is not ranked 3rd') corresponds to one of the precursors of Sen's condition mentioned in Section 1.

The first set of conditions ('is not ranked 1st') corresponds to single-cavedness for every triple. The general condition of single-cavedness requires the existence of a single ordering of all candidates from 'left'-most to 'right'-most such that each voter has a least preferred position on that 'left'/'right' ordering with increasing preference for candidates as they get increasingly distant from the least preferred position. Single-cavedness implies singlecavedness for every triple, but not vice-versa.

Single-cavedness. There exists a bijection $\Omega:\left\{x_{1}, x_{2}, \ldots, x_{k}\right\} \rightarrow\{1,2, \ldots, k\}$ such that, for every triple of candidates $x_{1}, x_{2}, x_{3}$ and every voter $i$, if $\left(\Omega\left(x_{1}\right)<\Omega\left(x_{2}\right)<\Omega\left(x_{3}\right)\right)$ or $\left(\Omega\left(x_{3}\right)<\Omega\left(x_{2}\right)<\Omega\left(x_{1}\right)\right)$, then $\left[x_{2} \succ x_{1}\right.$ implies $\left.x_{3} \succ x_{2}\right]$ for voter $i$.

The bijection $\Omega$ represents the left-right ordering of the candidates, and the condition $\left(\Omega\left(x_{1}\right)<\Omega\left(x_{2}\right)<\Omega\left(x_{3}\right)\right)$ or $\left(\Omega\left(x_{3}\right)<\Omega\left(x_{2}\right)<\Omega\left(x_{1}\right)\right)$ means that $x_{2}$ is 'between' $x_{1}$ and $x_{3}$ with respect to that left-right ordering.

The weaker condition of single-cavedness for triples permits a different bijection $\Omega$ for every triple of candidates. For the purposes of avoiding cycles, the weaker condition is sufficient. 


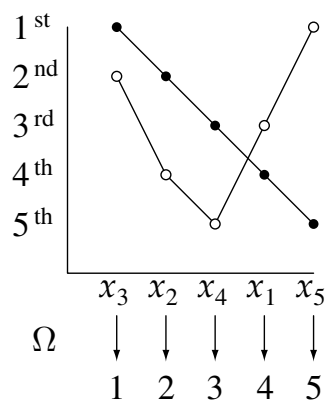

Fig. 1 Two orderings which are single-caved.

The second set of conditions ('is not ranked 2nd') corresponds to separability into two groups for every triple. The general condition of separability into two groups requires that any subset of the set of all candidates can be partitioned into two parts such that each voter prefers any candidate in one of the two parts to any candidate in the other. Separability into two groups implies separability into two groups for every triple, but not vice-versa.

Separability into two groups. Any subset $Y$ of the set of all candidates can be partitioned into two disjoint non-empty subsets $Y_{1}$ and $Y_{2}$ such that, for every voter $i$, either [for all $x_{1} \in Y_{1}$ and all $x_{2} \in Y_{2}, x_{1} \succ x_{2}$ ] or [for all $x_{1} \in Y_{1}$ and all $x_{2} \in Y_{2}, x_{2} \succ x_{1}$ ] for voter $i$.

Separability into two groups for every triple requires the existence of the required partition only for any triple of candidates.

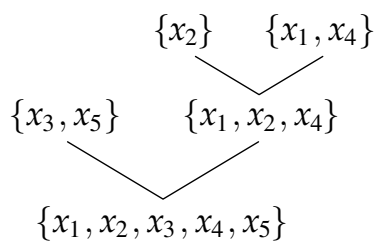

Fig. 2 Separability into two groups. For each vertex, each voter prefers any candidate in one branch to any candidate in the other.

The third set of conditions ('is not ranked 3rd') corresponds to single-peakedness for every triple. The general condition of single-peakedness requires the existence of a single ordering of all candidates from 'left'-most to 'right'-most such that each voter has a most preferred position on that 'left'/'right' ordering with decreasing preference for candidates as they get increasingly distant from the most preferred position. Single-peakedness implies single-peakedness for every triple, but not vice-versa. 


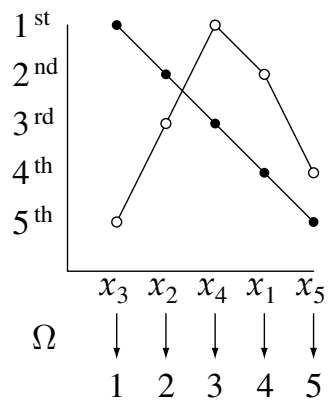

Fig. 3 Two orderings which are single-peaked.

Single-peakedness. There exists a bijection $\Omega:\left\{x_{1}, x_{2}, \ldots, x_{k}\right\} \rightarrow\{1,2, \ldots, k\}$ such that, for every triple of candidates $x_{1}, x_{2}, x_{3}$ and every voter $i$, if $\left(\Omega\left(x_{1}\right)<\Omega\left(x_{2}\right)<\right.$ $\left.\Omega\left(x_{3}\right)\right)$ or $\left(\Omega\left(x_{3}\right)<\Omega\left(x_{2}\right)<\Omega\left(x_{1}\right)\right)$, then $\left[x_{1} \succ x_{2}\right.$ implies $\left.x_{2} \succ x_{3}\right]$ for voter $i$.

The weaker condition of single-peakedness for triples permits a different bijection $\Omega$ for every triple of candidates. For the purposes of avoiding cycles, the weaker condition is sufficient.

\section{Proving Sen's result in full generality}

Finally, let us briefly sketch how our method can be used to allow the consideration of indifference in individual preference orderings, and thus to prove Sen's result in full generality. Allowing indifference means that there are three different ways in which a voter might rank two candidates, $x_{1}$ and $x_{2}$. The voter might rank one strictly above the other, i.e. $x_{1} \succ x_{2}$ or $x_{2} \succ x_{1}$; the voter might have an equal preference for both, i.e. $x_{1} \approx x_{2}$; or the voter might not rank or compare them at all. The first possibility corresponds to a strict ordering, the second to indifference, and the third to an incomplete ordering, between $x_{1}$ and $x_{2}$. Following Sen's result, we will only consider the first two possibilities and rule out incomplete orderings. In principle, however, incomplete orderings are also representable in terms of the matrix formalism introduced in Section 2.

Over triples of candidates, there are 7 possible preference orderings with indifference between two or more candidates, in addition to the 6 strict orderings shown above: $x_{1} \succ$ $x_{2} \approx x_{3}, x_{1} \approx x_{2} \succ x_{3}, x_{2} \succ x_{1} \approx x_{3}, x_{1} \approx x_{3} \succ x_{2}, x_{3} \succ x_{1} \approx x_{2}, x_{2} \approx x_{3} \succ x_{1}$, and $x_{1} \approx x_{2} \approx x_{3}$. (Note that $x_{1} \succ x_{2} \approx x_{3}$ and $x_{1} \succ x_{3} \approx x_{2}$ are considered the same.)

We define a corresponding notation. Here $a_{h i j}$ has the same interpretation as before. For weak orderings, let (for example) $a_{h \succ i \approx j}$ denote the number of voters holding the preference ordering $x_{h} \succ x_{i} \approx x_{j}$. The matrix corresponding to $x_{1} \succ x_{2} \approx x_{3}$, for example, is $M_{1 \succ 2 \approx 3}=\left(\begin{array}{lll}0 & 1 & 1 \\ 0 & 0 & 0 \\ 0 & 0 & 0\end{array}\right)$.

Strict orderings correspond to matrices with three non-zero entries, as defined in Section 2 , weak orderings correspond to matrices with two non-zero entries, except $x_{1} \approx x_{2} \approx x_{3}$, which corresponds to the matrix consisting only of zeros. 
In analogy to the argument above, pairwise majority voting corresponds to the following weighted sum:

$$
\begin{aligned}
S= & a_{123} M_{123}+a_{132} M_{132}+a_{213} M_{213}+a_{231} M_{231}+a_{312} M_{312}+a_{321} M_{321} \\
& +a_{1 \approx 2 \succ 3} M_{1 \approx 2 \succ 3}+a_{1 \approx 3 \succ 2} M_{1 \approx 3 \succ 2}+a_{2 \approx 3 \succ 1} M_{2 \approx 3 \succ 1} \\
& +a_{3 \succ 1 \approx 2} M_{3 \succ 1 \approx 2+a_{2 \succ 1 \approx 3} M_{2 \succ 1 \approx 3}+a_{1 \succ 2 \approx 3} M_{1 \succ 2 \approx 3}} \\
& =\left(\begin{array}{ccc}
a_{123}+a_{132}+a_{312}+a_{1 \approx 3 \succ 2}+a_{1 \succ 2 \approx 3} & a_{123}+a_{132}+a_{213}+a_{1 \approx 2 \succ 3}+a_{1 \succ 2 \approx 3} \\
a_{213}+a_{231}+a_{321}+a_{2 \approx 3 \succ 1}+a_{2 \succ 1 \approx 3} & 0 & a_{123}+a_{213}+a_{231}+a_{1 \approx 2 \succ 3}+a_{2 \succ 1 \approx 3} \\
a_{231}+a_{312}+a_{321}+a_{2 \approx 3 \succ 1}+a_{3 \succ 1 \approx 2} & a_{132}+a_{312}+a_{321}+a_{1 \approx 3 \succ 2}+a_{3 \succ 1 \approx 2} & 0
\end{array}\right)
\end{aligned}
$$

For simplicity, we assume that, for any pair of candidates $x_{1}, x_{2}$, the number of voters who do not have the preference ordering $x_{1} \approx x_{2}$ is odd. This assumption is different from Sen's. Sen's own assumption is that, for any triple of candidates $x_{1}, x_{2}, x_{3}$, the number of voters who do not have the preference ordering $x_{1} \approx x_{2} \approx x_{3}$ is odd. The following two examples show that Sen's assumption and our assumption are logically independent.

Case (i): Our assumption is satisfied, but Sen's is not. Consider 6 voters with the following preference orderings:
(1) $x_{1} \approx x_{2} \succ x_{3} \succ x_{4}$
(2) $x_{1} \approx x_{3} \succ x_{2} \succ x_{4}$
(3) $x_{1} \approx x_{4} \succ x_{2} \succ x_{3}$,
(4) $x_{2} \approx x_{3} \succ x_{1} \succ x_{4}$
(5) $x_{2} \approx x_{4} \succ x_{1} \succ x_{3}$,
(6) $x_{3} \approx x_{4} \succ x_{1} \succ x_{2}$.

Case (ii): Sen's assumption is satisfied, but ours is not. Consider 3 voters with the following preference orderings:

$$
\text { (1) } x_{1} \succ x_{2} \approx x_{3}, \quad \text { (2) } x_{2} \succ x_{3} \approx x_{1}, \quad \text { (3) } x_{3} \succ x_{1} \approx x_{2} \text {. }
$$

Our assumption has the possible advantage of being slightly easier to verify than Sen's. Verifying whether the number of voters who are not indifferent over a pair of candidates is odd is simpler than verifying whether the number who are not entirely indifferent over a triple of candidates (in the sense of holding the preference ordering $x_{1} \approx x_{2} \approx x_{3}$ ) is odd.

Given that the number of voters that are not indifferent about any particular pair of candidates is odd, there will not be any ties under majority voting, and, as before, the only two logically possible cycles are $x_{1} \succ x_{2} \succ x_{3} \succ x_{1}$ and $x_{1} \succ x_{3} \succ x_{2} \succ x_{1}$. The first cycle corresponds to the following inequalities:

$$
\begin{aligned}
& x_{1} \succ x_{2} \text { means } s_{12}>s_{21}, \text { i.e. } \\
& a_{123}+a_{132}+a_{312}+a_{1 \approx 3 \succ 2}+a_{1 \succ 2 \approx 3}>a_{213}+a_{231}+a_{321}+a_{2 \approx 3 \succ 1}+a_{2 \succ 1 \approx 3} . \text { (1) } \\
& x_{2} \succ x_{3} \text { means } s_{23}>s_{32}, \text { i.e. } \\
& a_{123}+a_{213}+a_{231}+a_{1 \approx 2 \succ 3}+a_{2 \succ 1 \approx 3}>a_{132}+a_{312}+a_{321}+a_{1 \approx 3 \succ 2}+a_{3 \succ 1 \approx 2 .} .(2) \\
& x_{3} \succ x_{1} \text { means } s_{31}>s_{13}, \text { i.e. } \\
& a_{231}+a_{312}+a_{321}+a_{2 \approx 3 \succ 1}+a_{3 \succ 1 \approx 2}>a_{123}+a_{132}+a_{213}+a_{1 \approx 2 \succ 3}+a_{1 \succ 2 \approx 3} . \text { (3) }
\end{aligned}
$$


Adding pairs of these inequalities leads to:
(1) $+(2)$ implies
$a_{123}+a_{1 \approx 2 \succ 3}+a_{1 \succ 2 \approx 3}>a_{321}+a_{2 \approx 3 \succ 1}+a_{3 \succ 1 \approx 2}$,
(1) + (3) implies
$a_{312}+a_{1 \approx 3 \succ 2}+a_{3 \succ 1 \approx 2}>a_{213}+a_{1 \approx 2 \succ 3}+a_{2 \succ 1 \approx 3}$,
(2) $+(3)$ implies
$a_{231}+a_{2 \approx 3 \succ 1}+a_{2 \succ 1 \approx 3}>a_{132}+a_{1 \approx 3 \succ 2}+a_{1 \succ 2 \approx 3}$.

Analogously, the second cycle implies the reverse inequalities. Using (a version of) Lemma 4 as before, we can state the following theorem:

Theorem 3 Suppose, for every triple of distinct candidates $x_{1}, x_{2}, x_{3}$, we have $\left(a_{123}+\right.$ $a_{1 \approx 2 \succ 3}+a_{1 \succ 2 \approx 3}=0$ or $a_{312}+a_{1 \approx 3 \succ 2}+a_{3 \succ 1 \approx 2}=0$ or $\left.a_{231}+a_{2 \approx 3 \succ 1}+a_{2 \succ 1 \approx 3}=0\right)$ and $\left(a_{321}+a_{2 \approx 3 \succ 1}+a_{3 \succ 1 \approx 2}=0\right.$ or $a_{213}+a_{1 \approx 2 \succ 3}+a_{2 \succ 1 \approx 3}=0$ or $a_{132}+a_{1 \approx 3 \succ 2}+a_{1 \succ 2 \approx 3}=$ 0 ). Then pairwise majority voting generates a transitive (hence acylic) majority preference ordering.

To see that the condition of Theorem 3 is equivalent to Sen's condition of triplewise valuerestriction, we just need to use the interpretation, in the case of indifference, that each candidate can have more than one rank within a voter's preference ordering, i.e. if $x_{h} \approx$ $x_{i} \succ x_{j}$, then $x_{h}$ and $x_{i}$ would each be regarded as both first and second within the given preference ordering among $x_{h}, x_{i}, x_{j}$. Using this interpretation and stating the condition of Theorem 3 in a form similar to Table 1 then yields a version of Sen's general result.

\section{A necessary and sufficient condition for avoiding cycles}

As we have pointed out, triplewise value-restriction is a sufficient condition for avoiding cycles. It is a generalization of its precursors, which are themselves sufficient conditions. But it is still not a necessary and sufficient condition. Can triplewise value-restriction itself be further generalized? How 'close' is it to a necessary and sufficient condition? We will now see that there is still some logical space between Sen's condition and a necessary and sufficient condition, but we suggest that this space may be too narrow to allow an appealing generalization of triplewise value-restriction.

We first state a necessary and sufficient condition for the occurrence of cycles. The result is a version of a result by Miller [3].

Theorem 4 Pairwise majority voting generates a cycle if and only if, for some triple of distinct candidates $x_{1}, x_{2}, x_{3}$, we have $\left(\left(a_{123}>a_{321}\right.\right.$ and $a_{312}>a_{213}$ and $\left.a_{231}>a_{132}\right)$ or $\left(a_{321}>a_{123}\right.$ and $a_{213}>a_{312}$ and $\left.\left.a_{132}>a_{231}\right)\right)$ and $\left|a_{123}-a_{321}\right|<\frac{n^{\prime}}{2}$ and $\left|a_{231}-a_{132}\right|<$ $\frac{n^{\prime}}{2}$ and $\left|a_{312}-a_{213}\right|<\frac{n^{\prime}}{2}$, where $n^{\prime}:=\left|a_{123}-a_{321}\right|+\left|a_{231}-a_{132}\right|+\left|a_{312}-a_{213}\right|$.

A proof is given in an appendix. Negating both sides of the if-and-only-if equivalence yields the following corollary of Theorem 4:

Corollary Pairwise majority voting generates a transitive (hence acylic) majority preference ordering if and only if, for every triple of distinct candidates $x_{1}, x_{2}, x_{3}$, we have $\left(\left(a_{123} \leq a_{321}\right.\right.$ or $a_{312} \leq a_{213}$ or $\left.a_{231} \leq a_{132}\right)$ and $\left(a_{321} \leq a_{123}\right.$ or $a_{213} \leq a_{312}$ or $\left.\left.a_{132} \leq a_{231}\right)\right)$ or $\left|a_{123}-a_{321}\right| \geq \frac{n^{\prime}}{2}$ or $\left|a_{231}-a_{132}\right| \geq \frac{n^{\prime}}{2}$ or $\left|a_{312}-a_{213}\right| \geq \frac{n^{\prime}}{2}$, where $n^{\prime}:=\left|a_{123}-a_{321}\right|+\left|a_{231}-a_{132}\right|+\left|a_{312}-a_{213}\right|$. 
Sen's condition implies, but is not implied by, the condition of the corollary of Theorem 4 . However, to see why it may nonetheless be impossible to find an appealing generalization of Sen's condition, let us introduce a criterion for describing a condition on a profile as simple. A condition (on a profile) is simple if it is dependent, for each logically possible preference ordering, only on whether or not that ordering occurs in the profile, but not on the number of voters holding the given ordering. Thus a condition is simple if it consists only of propositions of the forms $a_{h i j}=0$ and $a_{h i j} \neq 0$, as well as conjunctions or disjunctions of such propositions. The condition of triplewise value-restriction as defined in Section 2 satisfies the criterion of simplicity (leaving aside the requirement that $n$ be odd). Technically, triplewise value-restriction in its full generality already violates the criterion, as it requires the number of voters who are not indifferent about any particular triple (or pair) of alternatives to be odd.

The task of finding a simple sufficient condition for the avoidance of cycles, then, is to find a condition with the following two properties:

(i) the condition's basic components are only propositions of the forms $a_{h i j}=0$ and $a_{h i j} \neq 0$;

(ii) the condition implies

$$
\begin{gathered}
\left(\left(a_{123} \leq a_{321} \text { or } a_{312} \leq a_{213} \text { or } a_{231} \leq a_{132}\right)\right. \\
\text { and } \left.\left(a_{321} \leq a_{123} \text { or } a_{213} \leq a_{312} \text { or } a_{132} \leq a_{231}\right)\right) \\
\text { or }\left|a_{123}-a_{321}\right| \geq \frac{n^{\prime}}{2} \text { or }\left|a_{231}-a_{132}\right| \geq \frac{n^{\prime}}{2} \text { or }\left|a_{312}-a_{213}\right| \geq \frac{n^{\prime}}{2}, \\
\text { where } n^{\prime}:=\left|a_{123}-a_{321}\right|+\left|a_{231}-a_{132}\right|+\left|a_{312}-a_{213}\right| .
\end{gathered}
$$

Sen's solution, namely $\left(a_{123}=0\right.$ or $a_{312}=0$ or $\left.a_{231}=0\right)$ and $\left(a_{132}=0\right.$ or $a_{321}=0$ or $\left.a_{213}=0\right)$, seems to be the most general one we can get.

\section{Appendix: Proof of Theorem 4}

Step 1 . Let $x_{1}, x_{2}, x_{3}$ be any triple of candidates.

There is a majority cycle of type $x_{1} \succ x_{2} \succ x_{3} \succ x_{1}$

$\Leftrightarrow$

$\Rightarrow \quad\left(a_{123}-a_{321}+a_{231}\left(a_{231}+a_{312}+a_{321}>a_{123}+a_{132}+a_{213}\right)\right.$

and $\left(a_{123}-a_{321}+a_{231}-a_{132}+a_{312}-a_{213}>2\left(a_{312}-a_{213}\right)\right)$

and $\left(a_{123}-a_{321}+a_{231}-a_{132}+a_{312}-a_{213}>2\left(a_{123}-a_{321}\right)\right)$.

Similarly,

$$
\begin{array}{r}
\text { there is a majority cycle of type } x_{1} \succ x_{3} \succ x_{2} \succ x_{1} \\
\Leftrightarrow \quad \begin{aligned}
\left(a_{321}-a_{123}+a_{132}-a_{231}+a_{213}-a_{312}\right. & \left.>2\left(a_{132}-a_{231}\right)\right) \\
\text { and }\left(a_{321}-a_{123}+a_{132}-a_{231}+a_{213}-a_{312}\right. & \left.>2\left(a_{213}-a_{312}\right)\right) \\
\text { and }\left(a_{321}-a_{123}+a_{132}-a_{231}+a_{213}-a_{312}\right. & \left.>2\left(a_{321}-a_{123}\right)\right) .
\end{aligned}
\end{array}
$$


Step 2. Suppose there is some majority cycle. By Lemma 4, this implies that there is a cycle over three candidates, say $x_{1}, x_{2}, x_{3}$. The cycle must be of Type $1\left(x_{1} \succ x_{2} \succ x_{3} \succ x_{1}\right)$ or of Type $2\left(x_{1} \succ x_{3} \succ x_{2} \succ x_{1}\right)$. As we have seen in Section 2, a cycle of Type 1 implies $\left(a_{123}>a_{321}\right.$ and $a_{312}>a_{213}$ and $\left.a_{231}>a_{132}\right)$. By Step 1, we then also have

$$
\begin{aligned}
& \quad\left(\left|a_{123}-a_{321}\right|+\left|a_{231}-a_{132}\right|+\left|a_{312}-a_{213}\right|>2\left|a_{231}-a_{132}\right|\right) \\
& \text { and }\left(\left|a_{123}-a_{321}\right|+\left|a_{231}-a_{132}\right|+\left|a_{312}-a_{213}\right|>2\left|a_{312}-a_{213}\right|\right) \\
& \text { and }\left(\left|a_{123}-a_{321}\right|+\left|a_{231}-a_{132}\right|+\left|a_{312}-a_{213}\right|>2\left|a_{123}-a_{321}\right|\right) .
\end{aligned}
$$

Also, as we have seen in Section 2, a cycle of Type 2 implies $\left(a_{321}>a_{123}\right.$ and $a_{213}>a_{312}$ and $\left.a_{132}>a_{231}\right)$. By Step 1, we then also have

$$
\begin{aligned}
& \qquad\left(\left|a_{123}-a_{321}\right|+\left|a_{231}-a_{132}\right|+\left|a_{312}-a_{213}\right|>2\left|a_{231}-a_{132}\right|\right) \\
& \text { and }\left(\left|a_{123}-a_{321}\right|+\left|a_{231}-a_{132}\right|+\left|a_{312}-a_{213}\right|>2\left|a_{312}-a_{213}\right|\right) \\
& \text { and }\left(\left|a_{123}-a_{321}\right|+\left|a_{231}-a_{132}\right|+\left|a_{312}-a_{213}\right|>2\left|a_{123}-a_{321}\right|\right) .
\end{aligned}
$$

Recall that $n^{\prime}=\left|a_{123}-a_{321}\right|+\left|a_{231}-a_{132}\right|+\left|a_{312}-a_{213}\right|$. Hence a majority cycle implies the following condition:

$$
\begin{aligned}
& \left(\left(a_{123}>a_{321} \text { and } a_{312}>a_{213} \text { and } a_{231}>a_{132}\right)\right. \\
& \text { or } \left.\left(a_{321}>a_{123} \text { and } a_{213}>a_{312} \text { and } a_{132}>a_{231}\right)\right) \\
& \text { and }\left|a_{123}-a_{321}\right|<\frac{n^{\prime}}{2} \text { and }\left|a_{231}-a_{132}\right|<\frac{n^{\prime}}{2} \text { and }\left|a_{312}-a_{213}\right|<\frac{n^{\prime}}{2}
\end{aligned}
$$

Suppose, conversely, there exists a triple of candidates, $x_{1}, x_{2}, x_{3}$, such that $(*)$ holds. We must have either $\left(a_{123}>a_{321}\right.$ and $a_{312}>a_{213}$ and $\left.a_{231}>a_{132}\right)$ or $\left(a_{321}>a_{123}\right.$ and $a_{213}>a_{312}$ and $\left.a_{132}>a_{231}\right)$. If $\left(a_{123}>a_{321}\right.$ and $a_{312}>a_{213}$ and $\left.a_{231}>a_{132}\right)$, we have

$$
\begin{array}{r}
\left(a_{123}-a_{321}+a_{231}-a_{132}+a_{312}-a_{213}>2\left(a_{231}-a_{132}\right)\right) \\
\text { and }\left(a_{123}-a_{321}+a_{231}-a_{132}+a_{312}-a_{213}>2\left(a_{312}-a_{213}\right)\right) \\
\text { and }\left(a_{123}-a_{321}+a_{231}-a_{132}+a_{312}-a_{213}>2\left(a_{123}-a_{321}\right)\right),
\end{array}
$$

which implies a majority cycle of Type 1 , by Step 1 .

If $\left(a_{321}>a_{123}\right.$ and $a_{213}>a_{312}$ and $\left.a_{132}>a_{231}\right)$, we have

$$
\begin{array}{r}
\left(a_{321}-a_{123}+a_{132}-a_{231}+a_{213}-a_{312}>2\left(a_{132}-a_{231}\right)\right) \\
\text { and }\left(a_{321}-a_{123}+a_{132}-a_{231}+a_{213}-a_{312}>2\left(a_{213}-a_{312}\right)\right) \\
\text { and }\left(a_{321}-a_{123}+a_{132}-a_{231}+a_{213}-a_{312}>2\left(a_{321}-a_{123}\right)\right),
\end{array}
$$

which implies a majority cycle of Type 2, by Step 1 . 


\section{References}

[1] Black, D.: On the Rationale of Group Decision-Making. J. Political Economy 56 (1948), 23-34.

[2] Inada, K.: A Note on the Simple Majority Decision Rule, Econometrica 32 (1964), 525-531.

[3] Miller, N.: The Geometry of Voting Cycles: Theoretical Developments. Paper presented at the Workshop on Mathematical Models of Individual and Public Choice, Institute for Mathematical Behavioral Sciences, University of California, Irvine, July 2000.

[4] Riker, W.H.: Liberalism Against Populism. W.H. Freeman, San Franscisco 1982.

[5] Sen, A.K.: A Possibility Theorem on Majority Decisions. Econometrica 34 (1966), 491-499, reprinted in Sen, A.K.: Choice, Welfare and Measurement. Blackwell, Oxford 1982.

[6] Ward, B.: Majority Voting and Alternative Forms of Public Enterprises. In: Margolis, J. (ed.): The Public Economy of Urban Communities. Johns Hopkins Press, Baltimore 1965.

Christian Elsholtz

Department of Mathematics

Royal Holloway University of London

Egham

Surrey TW20 0EX, U.K.

e-mail: christian.elsholtz@rhul.ac.uk

Christian List

Department of Government

London School of Economics

Houghton Street

London WC2A 2AE, U.K.

e-mail: c.list@lse.ac.uk 